\title{
Expansion and Age of the X-Ray Synchrotron-dominated Supernova Remnant G330.2+1.0
}

\author{
Kazimierz J. Borkowski ${ }^{1}$, Stephen P. Reynolds ${ }^{1}$ (D), Brian J. Williams ${ }^{2}$ (D), and Robert Petre ${ }^{2}$ (D) \\ ${ }^{1}$ Department of Physics, North Carolina State University, Raleigh, NC 27695-8202, USA; kborkow@ncsu.edu \\ ${ }^{2}$ NASA/Goddard Space Flight Center, Greenbelt, MD 20771, USA \\ Received 2018 August 25; revised 2018 October 23; accepted 2018 October 29; published 2018 November 20
}

\begin{abstract}
We report new Chandra observations of one of the few Galactic supernova remnants whose X-ray spectrum is dominated by nonthermal synchrotron radiation, G330.2+1.0. We find that between 2006 and 2017, some parts of the shell have expanded by about $1 \%$, giving a free-expansion (undecelerated) age of about $1000 \mathrm{yr}$, and implying shock velocities there of $9000 \mathrm{~km} \mathrm{~s}^{-1}$ for a distance of $5 \mathrm{kpc}$. Somewhat slower expansion is seen elsewhere around the remnant periphery, in particular in compact knots. Because some deceleration must have taken place, we infer that G330.2+1.0 is less than about $1000 \mathrm{yr}$ old. Thus, G330.2+1.0 is one of only four Galactic core-collapse remnants of the last millennium. The large size, low brightness, and young age require a very low ambient density, suggesting expansion in a stellar-wind bubble. We suggest that in the east, where some thermal emission is seen and expansion velocities are much slower, the shock has reached the edge of the cavity. The high shock velocities can easily accelerate relativistic electrons to X-ray-emitting energies. A few small regions show highly significant brightness changes by $10 \%-20 \%$, both brightening and fading, a phenomenon previously observed in only two supernova remnants, indicating strong and/or turbulent magnetic fields.
\end{abstract}

Key words: ISM: individual objects (G330.2+1.0) - ISM: supernova remnants - X-rays: ISM

\section{Introduction}

The fast shocks in young supernova remnants (SNRs) accelerate particles to high enough energies to emit synchrotron radiation from radio to X-ray wavelengths. Studying such remnants can provide information on the detailed physics of the acceleration process. In a few remnants, synchrotron X-rays are not only present, but dominate the spectrum (see Reynolds 2008 for a review). While several are quite well known, one lesserknown object has received recent attention, with a dominant synchrotron spectrum, thermal emission in a few locations, and a compact central object (CCO), presumably a neutron star: G330.2+1.0 (Park et al. 2006, 2009; Torii et al. 2006).

At radio wavelengths, G330.2+1.0 has a distorted shell morphology with a much brighter, confused region to the east (Caswell et al. 1983). In X-rays (Figure 1), the shell is clearer, coinciding fairly well with much of the faint radio shell.

G330.2+1.0 was observed with Chandra (Park et al. 2009) for $50 \mathrm{ks}$ in 2006, and with XMM-Newton in 2008 (Park et al. 2009, with an effective exposure $33 \mathrm{ks}$ ) and 2015 (Williams et al. 2018, hereafter W18, with effective exposure of about $90 \mathrm{ks}$ ). Line emission is virtually absent except in a region in the east near the radio maximum. For the rest of the remnant, the X-ray spectrum between 1 and $7 \mathrm{keV}$ can be well described by a single power law of photon index $\Gamma \sim 2.3$ (Park et al. 2009; W18), with little variation across the remnant, including not only the clear shell, but emission from the faint interior (W18). These values are typical for power-law components seen in other SNRs with nonthermal X-ray emission (e.g., RX J1713.7-3946; Katsuda et al. 2015). However, the spectrum could also be well described with a simple model of synchrotron emission from a power-law energy distribution of electrons with an exponential cutoff at some energy $E_{m}$ (model srcut in XSPEC; Reynolds \& Keohane 1999). The photon frequency corresponding to $E_{m}$ was found to be around $2 \times 10^{17} \mathrm{~Hz}$, which is quite a high value; only the youngest Galactic SNR G1.9+0.3 has a higher one, of about $5 \times 10^{17} \mathrm{~Hz}$ (Reynolds et al. 2009).

$\mathrm{H}$ I absorption observations give a minimum distance of $4.9 \mathrm{kpc}$ (McClure-Griffiths et al. 2001), the distance to some $\mathrm{H}$ I emission regions with which it might be interacting. While a distance as large as $10 \mathrm{kpc}$ cannot be ruled out, it would imply unlikely properties such as an age of $4000 \mathrm{yr}$ for a synchrotron-dominated SNR, and a $z$-distance of 200 pc above the Galactic plane for a core-collapse SNR. So we take a distance of $5 d_{5} \mathrm{kpc}$, at which the mean angular radius of about $5^{\prime}$ corresponds to a linear radius of $7.3 d_{5}$ pc. W18 use the thermal fits to the east region to obtain an emission measure and an estimate of the preshock density in that region of about $n_{0} \sim 1 d_{5}^{-1 / 2} \mathrm{~cm}^{-3}$, but the absence of thermal emission elsewhere suggests that this value is higher than typical for the remnant environment. W18 find an electron temperature $T_{e}=0.46(0.40,0.58) \mathrm{keV}$ for a shock velocity of order 650 $\mathrm{km} \mathrm{s}^{-1}$; again, this is unlikely to typify the remnant as a whole.

\section{Observations and Expansion Measurement Methods}

Chandra observed G330.2+1.0 again in two $74.1 \mathrm{ks}$ exposures with the Advanced Charge-coupled Device (CCD) Imaging Spectrometer (ACIS) I array on 2017 May 2 and 5, in Very Faint mode. We used CIAO version 4.9 and CALDB version 4.7.8 to reprocess these observations, align them using the centrally located CCO, and finally merge them together. No particle flares were found. The total exposure time is $148.3 \mathrm{ks}$. A smoothed X-ray image, extracted from merged and smoothed Chandra datacubes, is shown in Figure 1. The remnant's faintness forces us to select only those emission features that are bright and sharp enough to measure expansion reliably (see Figures 2 and 3 for their location within the remnant).

The first-epoch Chandra observations of G330.2+1.0 in 2006, also using the ACIS-I array in the Very Faint mode, were 


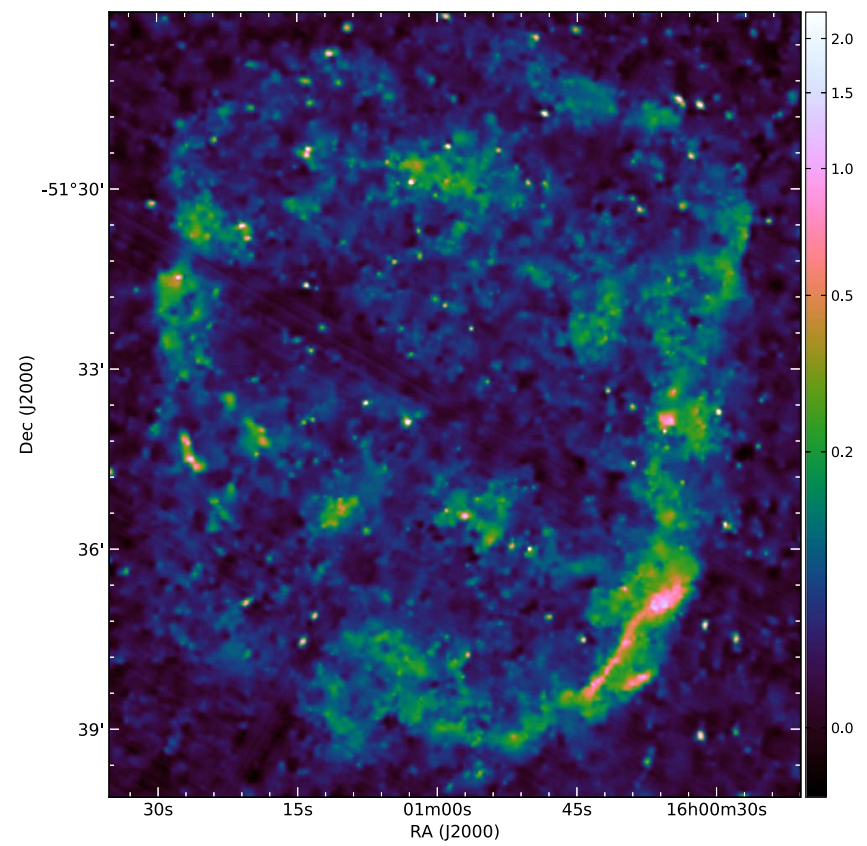

Figure 1. Smoothed background-subtracted Chandra image of G330.2+1.0 $(0.6-7.1 \mathrm{keV})$. The blast wave extends farthest away from the centrally located CCO toward the northwest. The bright southwest rim is inward facing. Compact emission knots are seen to the east and west of the CCO. The scale is in counts per $0.769 \times 0$. 769 pixel. Intensities are shown with the cubehelix color scheme of Green (2011). The CCO is the bright point source near the center.

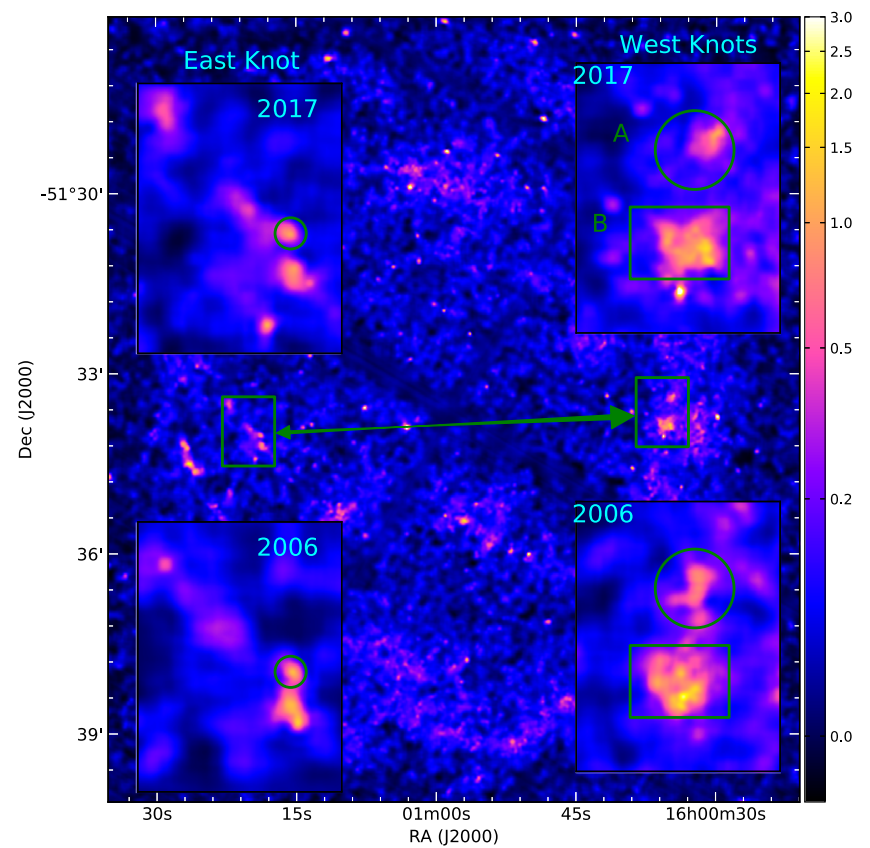

Figure 2. Zoom-ins of two regions (in green boxes on opposite sides of the $\mathrm{CCO})$ containing compact emission knots. Motions of two knots in the west (West Knots A and B), and at least one knot in the east (East Knot) are apparent between 2006 and 2017 (bottom and top insets, respectively). The scale is in counts per 0 . $769 \times 0$. 769 pixel.

reprocessed in the same way as the second epoch observations. The exposure time is much shorter $(50 \mathrm{ks})$, so the uncertainties in our expansion measurements are almost entirely due to the poor signal-to-noise ratio $(\mathrm{S} / \mathrm{N})$ of the first-epoch observations. The CCO was used for inter-epoch alignment, but a point
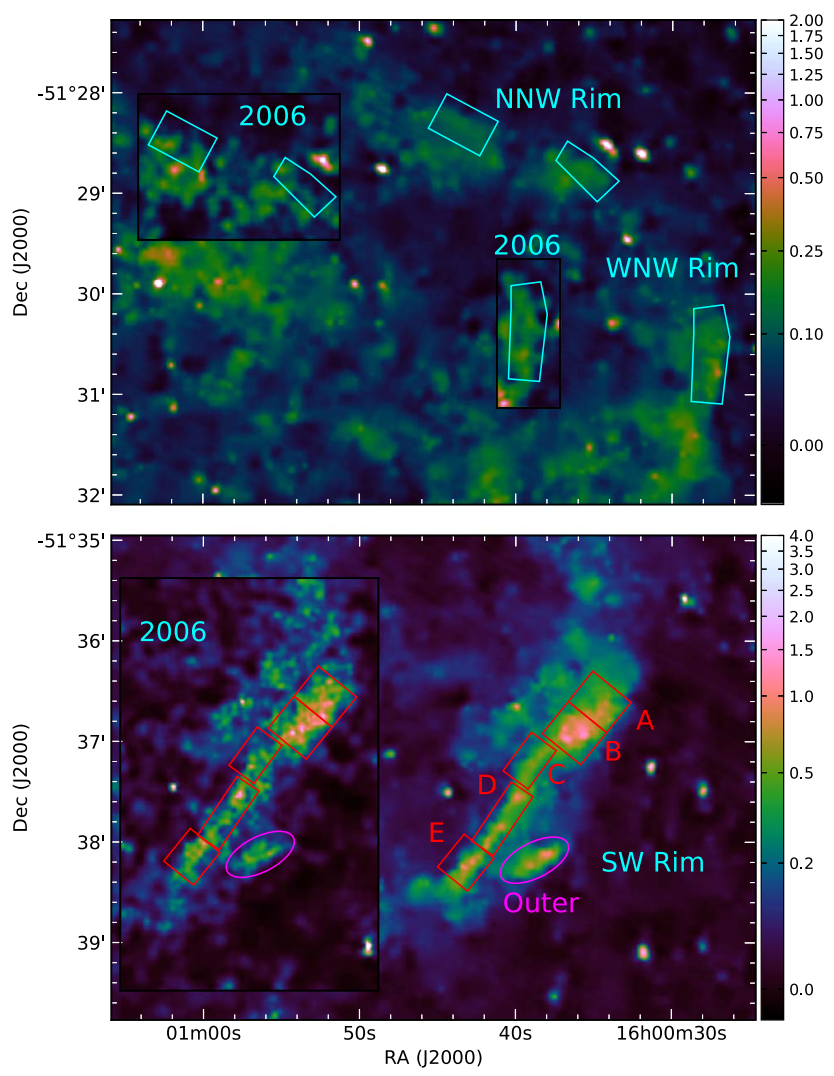

Figure 3. Zoom-ins of northwest (top) and southwest (bottom) rims of G330.2 +1.0 , with regions used for expansion measurements of the blast wave overlaid. Insets show these regions in 2006. The scale is in the same units as in Figure 1 (but insets are not to this scale).

source reference frame was also examined, in both cases using the same techniques as in our expansion measurements of the 500 yr old pulsar wind nebula in the SNR Kes 75 (Reynolds et al. 2018, hereafter R18). There are 18 sources with off-axis angle $\theta_{\mathrm{OAA}}<7^{\prime}$, encompassing our selected emission features, that are suitable for the astrometric alignment. Our null hypothesis is that their displacements between the two epochs, measured after alignment to the $\mathrm{CCO}$ and normalized by $1 \sigma$ errors estimated as in R18, satisfy the Rayleigh distribution that is expected if the alignment were perfect. The KolmogorovSmirnov test fails to reject this hypothesis with $82 \%$ confidence. The $\mathrm{CCO}$ and point source reference frames are displaced by only 77 mas when measured using point sources with $\theta_{\mathrm{OAA}}<5^{\prime}$ where our source position measurements are most reliable. The estimated (as in R18) positional uncertainties for the CCO are only slightly smaller, about 60 mas (at $90 \%$ confidence) for each pointing. Therefore, we find no evidence for misalignment of point sources or a discernible CCO motion.

The time baseline between the 2006 and 2017 observations is $10.95 \mathrm{yr}$, long enough to reliably measure expansion of most of the selected emission features with a variation of the method used by R18. First, we extracted a datacube from the merged 2017 data, with $1024^{2}$ image pixels and 16 spectral channels, in the energy range from 0.6 to $7.1 \mathrm{keV}$. The spatial pixel size is 0 ". $769 \times 0$ ". 769 (slightly more than $3 / 2$ of an ACIS 0 " 492 pixel). We smoothed this datacube with the multiscale partitioning method of Krishnamurthy et al. (2010), and then extracted smoothed images. Three relatively high-surfacebrightness emission knots (Figure 2), one in the east and two in the west, are significantly more compact than more diffuse 
Table 1

Expansion between 2006 and 2017

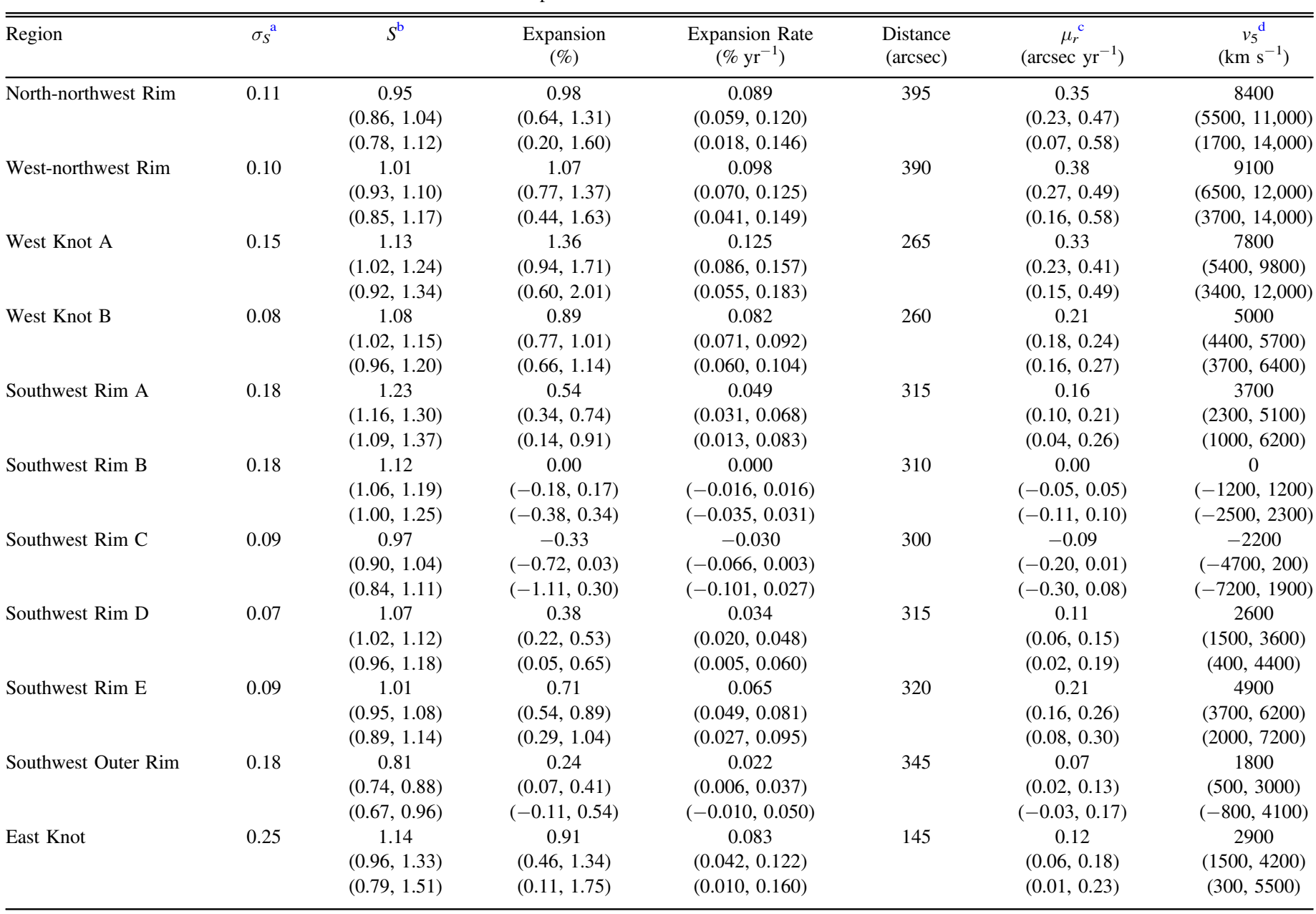

Notes. Credible intervals are two sets of $(16 \%, 84 \%)$ and $(2.5 \%, 97.5 \%)$ quantiles from MCMC draws.

${ }^{\mathrm{a}}$ Width of Gaussian prior for the surface brightness scaling factor $S$ (its mean was set to unity).

${ }^{\mathrm{b}}$ Surface brightness scaling factor.

${ }^{c}$ Radial proper motion.

d Expansion velocity is $v_{5} d_{5}$, with $d_{5}$ the distance in units of $5 \mathrm{kpc}$.

rims and filaments in north-northwest, west-northwest, and southwest (Figure 3). The East Knot is the most compact (FWHM of 2!"9). In order to avoid oversmoothing this and the other two knots, the penalty parameter that controls smoothing was set to 0.03 in this case (the smoothed image is shown in Figure 2) versus 0.06 for the rest of G330.2+1.0 (Figures 1 and 3 ). (Oversmoothing of the knots using the penalty parameter of 0.06 simply degrades our measurements; e.g., the expansion variance for the East Knot increases by over $60 \%$ without any significant change in the measured expansion.) After particle background subtraction and normalization by a monochromatic $(E=2.3 \mathrm{keV})$ exposure map, these smoothed images are used as models for the brightness distribution of the selected emission features of interest.

The smoothed 2017 models are fit to the unsmoothed 0.6-7.1 keV image from 2006 using Markov chain Monte Carlo (MCMC) methods as implemented in the PyMC software package (Patil et al. 2010). (But we show parts of a smoothed 2006 image in Figures 2 and 3 instead of the unsmoothed image - the penalty parameter was set to 0.02 in smoothing the 2006 datacube from which this smoothed image was extracted.)
Poisson statistics are assumed. In our MCMC simulations, we allow for changes in the physical image scale and in the surface-brightness scale factor $S$. Expansion is centered on the CCO. Spatial variations in the effective exposure time are accounted for by the monochromatic $(E=2.3 \mathrm{keV})$ exposure map. The particle background is modeled as described in Bartalucci et al. (2014). A uniform prior is assumed for expansion, reflecting our prior (lack of) knowledge about the age and dynamics of G330.2+1.0.

Approximately, the scale factor $S$ can be considered as a proxy for the (scaled) photon flux within each of our regions. We use a Gaussian prior for $S$ with mean of unity and variance $\sigma_{S}^{2}$. The lower bound to $\sigma_{S}$ is $\left(N_{s}+N_{b}\right)^{1 / 2} / N_{s}$ (where $N_{s}$ and $N_{b}$ are the total number of source and background counts within each of our regions in 2017). We conservatively assume the variance $\sigma_{S}^{2}$ to be twice as large, equal to $2\left(N_{s}+N_{b}\right) / N_{s}^{2}$. But for three regions in the southwest that unexpectedly showed evidence for apparent flux variations between the two epochs, Southwest Rims A and B, and Southwest Outer Rim, we further relaxed the prior for $S$ by setting $\sigma_{S}^{-2}$ to 30 . We list $\sigma_{S}$ estimated in this way in the second column of Table 1. 
For each region, our MCMC simulations involved about 15 chains, each 4000 iterations in length. We list chain-averaged means for expansion (and expansion rate) and $S$ in Table 1. Approximate radial distances measured from images, together with radial proper motions and velocities, are also listed there. Two sets of credible intervals are provided, corresponding to $(16 \%, 84 \%)$ and $(2.5 \%, 97.5 \%)$ quantiles from MCMC draws. Unless noted otherwise, we refer to the first set (corresponding to $1 \sigma$ errors) when quoting errors on expansion, $S$, and on other derived quantities.

\section{Results and Discussion}

G330.2+1.0 shows a highly irregular blast wave and a number of compact emission knots to the east and west of the CCO (Figure 1). We highlight three such emission knots in Figure 2, West Knots A and B, and the East Knot. They have contrasting spectral properties, with West Knots having hard spectra typical of emission in the western part of G330.2+1.0, thus being most prominent in the hard (2-7 keV) energy band in Figure 1 of W18 (see their region 10). The East Knot is in the inner part of a region labeled as "therm" in this Figure, within the most distinct knot of thermal emission there that is very prominent in the $1.2-2 \mathrm{keV}$ band, so its spectrum is presumably thermal. All three knots are moving away from the CCO with comparable expansion rates: $0.125_{-0.039}^{+0.032} \% \mathrm{yr}^{-1}$ and $0.082 \% \pm 0.011 \% \mathrm{yr}^{-1}$ for the West Knots $\mathrm{A}$ and $\mathrm{B}$, and $0.083_{-0.041}^{+0.039} \% \mathrm{yr}^{-1}$ for the East Knot (Table 1). We identify the East Knot with a dense clump of supernova (SN) ejecta because of its compactness, soft spectrum, and high transverse motion of $2900_{-1400}^{+1400} d_{5} \mathrm{~km} \mathrm{~s}^{-1}$. The West Knots might also be associated with fast-moving ejecta clumps, although their densities are probably much lower. Because their velocities are much higher, $5000_{-700}^{+700} d_{5} \mathrm{~km} \mathrm{~s}^{-1}$ for the West Knot B and possibly even more for the West Knot A, nonthermal emission is likely to be more efficiently produced, perhaps accounting for the profound differences in X-ray spectral properties between the East and West Knots.

The East and West Knots are located on opposite sides of the $\mathrm{CCO}$. This allows us to relax our approximation that the $\mathrm{CCO}$ has not moved away from the SN explosion site, by allowing these knots to expand uniformly around a common expansion center that may not coincide with the CCO. A 2D Gaussian prior with the FWHM of $25^{\prime \prime}$ centered on the CCO was used for the expansion center. Its width was chosen to exclude unrealistically large $\left(>30^{\prime \prime}\right)$ displacements produced by a hypothetical neutron star with age longer than $1500 \mathrm{yr}$ that is moving with the transverse velocity of $500 d_{5} \mathrm{~km} \mathrm{~s}^{-1}$ or more. We obtain an expansion rate of $0.085_{-0.010}^{+0.011} \% \mathrm{yr}^{-1}$, virtually identical to $0.083_{-0.009}^{+0.010} \% \mathrm{yr}^{-1}$ obtained with the expansion centered on the CCO. Allowing for the CCO motion results in only a modest loss in precision, and a statistically insignificant increase in expansion.

The strongly nonspherical blast wave extends farthest away from the CCO toward the northwest, with sharp rims visible in the north-northwest and west-northwest (upper panel in Figure 3). While a chip gap divides the north-northwest rim, expansion was measured by combining the two apparently noncontiguous rim sections. We find expansion rates of $0.089 \% \pm 0.031 \% \mathrm{yr}^{-1}$ and $0.098_{-0.028}^{-0.027} \% \mathrm{yr}^{-1}$ in north-northwest and west-northwest, virtually identical within errors (the weighted expansion rate is $0.094 \% \mathrm{yr}^{-1}$ ), and also comparable to the expansion found for the ejecta knots in the east and west

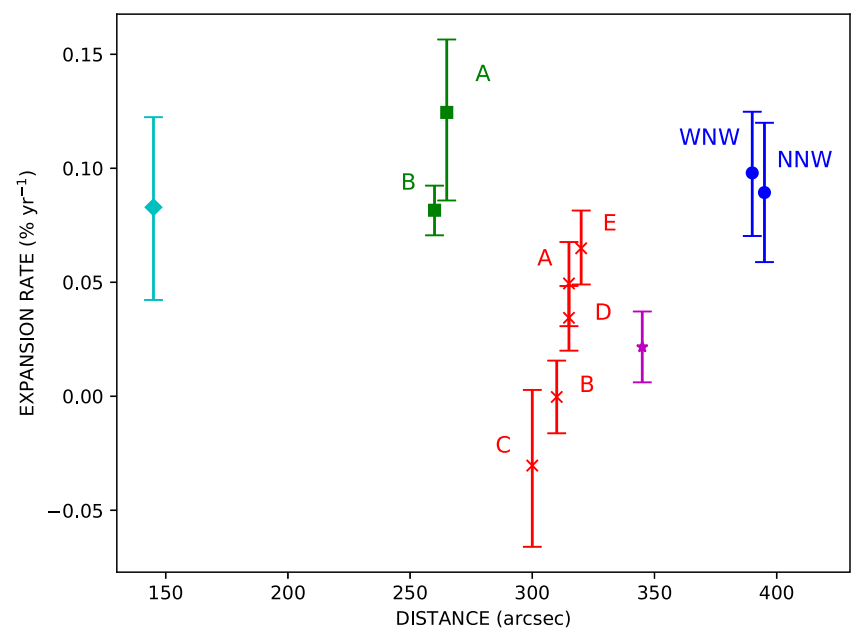

Figure 4. Expansion rate vs. radial distance from the CCO. From left to right: East Knot (cyan diamond), West Knots (green squares), Southwest Rim (red $\times$ 's), Southwest Outer Rim (magenta star) and West-northwest and Northnorthwest Rims (blue circles). A large range in expansion is apparent in the southwest.

(Figure 4). But the blast wave velocities of $9100_{-2600}^{+2500} d_{5} \mathrm{~km} \mathrm{~s}^{-1}$ in west-northwest and $8400_{-2900}^{+2900} d_{5} \mathrm{~km} \mathrm{~s}^{-1}$ in north-northwest are much larger than velocities of the compact knots.

The bright rim in the southwest of G330.2+1.0 has a complex morphology, with an inward-facing rim and a detached outer section that is perhaps not physically related. Figure 3 (lower panel) shows five regions spanning the entire bright inward-facing rim and an additional outer region where we measured radial expansion. We found no measurable motions near the apex of the Southwest Rim closest to the $\mathrm{CCO}$, but a significant expansion is detected on either side of it (Table 1 and Figure 4). The fastest expansion is found at the northwest and southeast tips of the Southwest Rim (labeled A and $\mathrm{E}$ in Figure 3): $0.049_{-0.019}^{+0.018} \% \mathrm{yr}^{-1}$ and $0.065_{-0.016}^{+0.017} \% \mathrm{yr}^{-1}$, respectively. This is still less than in the northwest or for the ejecta knots, but the radial velocity of $4900_{-1200}^{+1300} d_{5} \mathrm{~km} \mathrm{~s}^{-1}$ at its southeast tip is still impressive.

The scale factor $S$ is significantly above unity in the Southwest Rim northwest of its apex, and below unity for the Southwest Outer Rim (Table 1). The former includes the brightest part of G330.2+1.0, the Southwest Rim B, that faded by $12 \%$. Even more pronounced $(23 \%)$ fading is found for the adjacent Southwest Rim A, while the Southwest Outer Rim brightened by a comparable (19\%) amount. Such large flux variations cannot be accounted for by either statistical errors (see Table 1) or by systematic calibration errors that can reach only about $3 \%^{3}$ for a highly absorbed source such as G330.2 +1.0 . X-ray flux variations of any kind are rare in shell SNRs. Small-scale variations have been reported in the synchrotrondominated shell remnant G347.3-0.5 (RX J1713.7-3946; Uchiyama et al. 2007) while SN 1006, also synchrotrondominated, shows no such variations (Katsuda et al. 2010). Such variations seem to require magnetic fields strongly amplified in the shock waves (Uchiyama et al. 2007), but turbulent fields need not be as large on average (Bykov et al. 2008).

\footnotetext{
3 http://cxc.harvard.edu/cal/summary/Calibration_Status_Report. html\#ACIS_EA
} 
The very fast blast wave propagating through a low-density medium such as encountered in the northwest must have hit a dense obstacle in the southwest some time ago. The blast wave first arrived near the apex of the Southwest Rim, slowed down there, then started to wrap around this obstacle. Qualitatively, the observed expansion pattern and the overall geometry of the Southwest Rim are consistent with this scenario. Rapid brightness variations might also be expected for a relatively recent interaction.

Our expansion rates in the north and west of order $0.1 \% \mathrm{yr}^{-1}$ give an undecelerated age of $1000 \mathrm{yr}(800 \mathrm{yr}$ for the fastest knot, West Knot A). As some deceleration has almost certainly occurred, these are upper limits. Evidently G330.2+1.0 is one of the youngest SNRs in the Galaxy, probably younger even than the synchrotron-X-ray-dominated Type Ia remnant SN 1006. Along with Cas A, Kes 75 (R18), and the Crab, G330.2 +1.0 is one of the four youngest Galactic core-collapse remnants known. Even with the uncertain distance, the shock speeds that we measure are among the fastest ever measured for an SNR. Only G1.9+0.3, with speeds of order $14,000 \mathrm{~km} \mathrm{~s}^{-1}$ (Carlton et al. 2011), has significantly faster shocks.

The large average size $\left(7.3 d_{5} \mathrm{pc}\right)$, the young age of less than $1000 \mathrm{yr}$, very high shock speeds up to $9000 \mathrm{~km} \mathrm{~s}^{-1}$, and very low flux imply a very low mean density of ambient material that is typical of wind-blown bubbles around massive stars. However, the presence of thermal emission in the east quadrant indicates a considerably higher density there (W18). We suggest that G330.2+1.0 is expanding in an asymmetric cavity, likely produced by pre-supernova mass loss, but that in the east, the blast wave has reached the cavity wall and is expanding into much denser material. Then G330.2+1.0 is the first core-collapse SNR for which the interaction with a cavity wall has begun but is not complete, similar to the (probable) Type Ia remnant RCW 86 (Williams et al. 2011). A number of small-scale emission features are found in the east (Figure 1), suggesting a considerable amount of clumpiness within or near the cavity wall.

The high shock velocities that we find can easily account for the high rolloff frequencies of the synchrotron emission. If electron acceleration is limited by radiative losses, that frequency is independent of magnetic field: $\nu_{\text {rolloff }}=5 \times$ $10^{16} u_{8}^{2}\left(\eta R_{J}\left(\theta_{B n}\right)\right)^{-1} \mathrm{~Hz}$, where $u_{8}$ is the shock velocity in units of $10^{8} \mathrm{~cm} \mathrm{~s}^{-1}, \eta$ is the scattering mean free path of a relativistic electron in units of its gyroradius (the "gyrofactor"), and $R_{J}\left(\theta_{B n}\right)$ is a factor accounting for obliquity-dependence of shock acceleration (Reynolds 1998). For a strong, turbulent shock we expect $\eta R_{J} \sim 1$. The observed values of rolloff frequency, about $3 \times 10^{17} \mathrm{~Hz}$, then require only $u_{8} \gtrsim 3$, lower than most of the velocities that we measure. The shock velocities are so high that the age-limited maximum electron energy, which varies as $u_{8}^{4}$, is much higher than the loss-limited energy and hence not relevant.

W18 find no appreciable variation in rolloff frequencies among 14 positions around the rim, with uncertainties of order (60-80)\%, suggesting that unlike SN 1006, where systematic variations with azimuth of an order of magnitude are seen (Miceli et al. 2009) and attributed to systematic variations in shock obliquity, the upstream magnetic-field direction outside $\mathrm{G} 330.2+1.0$ is far from uniform.

\section{Conclusions}

We have measured the expansion of the X-ray-synchrotrondominated SNR G330.2+1.0 between 2006 and 2017. We find expansion rates of up to $0.12 \% \mathrm{yr}^{-1}$, for an undecelerated age of less than $1000 \mathrm{yr}$. Because some deceleration is certain to have occurred, the true age is even less. G330.2+1.0 is thus among the youngest core-collapse SNRs in the Galaxy, and has shock velocities faster than all known SNRs except the youngest Galactic SNR G1.9+0.3, thought to be Type Ia, whose X-rays are also dominated by synchrotron emission. The shock velocities in G330.2+1.0 range up to $9000 \mathrm{~km} \mathrm{~s}^{-1}$, in regions somewhat farther from the center than the average radius, where we presume the central neutron star to be the expansion center. Some distinct blobs have somewhat slower expansion rates, corresponding to speeds of about $3000-5000 \mathrm{~km} \mathrm{~s}^{-1}$, and softer spectra, suggestive of clumps of ejecta. Complex motions are seen in the southwest, where some regions have varied in brightness by $10 \%-20 \%$. The presence of thermal emission in one region in the east suggests interaction with much denser material there. We propose that G330.2+1.0 is expanding into a stellar-wind cavity and has reached the cavity wall in the east some time ago. A more recent impact with the cavity wall might have also occurred in the southwest in view of the strong blast wave deceleration and the presence of significant brightness changes there. Our age of order $1000 \mathrm{yr}$ or less may be significant for the cooling of the neutron star, which is evidently the youngest $\mathrm{CCO}$ after the $\mathrm{CCO}$ in $\mathrm{Cas} \mathrm{A}$.

We acknowledge support by NASA through Chandra General Observer Program grant SAO GO7-18051X.

Facility: CXO.

\section{ORCID iDs}

Stephen P. Reynolds (i) https://orcid.org/0000-00025365-5444

Brian J. Williams (iD https://orcid.org/0000-0003-2063-381X Robert Petre (1D https://orcid.org/0000-0003-3850-2041

\section{References}

Bartalucci, I., Mazzotta, P., Bourdin, H., \& Vikhlinin, A. 2014, A\&A, 566, A25

Bykov, A. M., Uvarov, Y. A., \& Ellison, D. C. 2008, ApJL, 689, L133

Carlton, A. K., Borkowski, K. J., Reynolds, S. P., et al. 2011, ApJL, 737, L22

Caswell, J. L., Haynes, R. F., Milne, D. K., \& Wellington, K. J. 1983, MNRAS, 204, 915

Green, D. A. 2011, BASI, 39, 289

Katsuda, S., Acero, F., Tominaga, N., et al. 2015, ApJ, 814, 29

Katsuda, S., Petre, R., Mori, K., et al. 2010, ApJ, 723, 383

Krishnamurthy, K., Raginsky, M., \& Willett, R. 2010, SIAM J. Imaging Sci., 3, 619

McClure-Griffiths, N. M., Green, A. J., Dickey, J. M., et al. 2001, ApJ, 551,394

Miceli, M., Bocchino, F., Iakubovskyi, D., et al. 2009, A\&A, 501, 239

Park, S., Kargaltsev, O., Pavlov, G. G., et al. 2009, ApJ, 695, 431

Park, S., Mori, K., Kargaltsev, O., et al. 2006, ApJL, 653, L37

Patil, A., Huard, D., \& Fonnesbeck, C. J. 2010, J. Stat. Software, 35, 4

Reynolds, S. P. 1998, ApJ, 493, 375

Reynolds, S. P. 2008, ARA\&A, 46, 89

Reynolds, S. P., Borkowski, K. J., Green, D. A., et al. 2009, ApJL, 695, L149 Reynolds, S. P., Borkowski, K. J., \& Gwynne, P. H. 2018, ApJ, 856, 133 Reynolds, S. P., \& Keohane, J. W. 1999, ApJ, 525, 368

Torii, K., Uchida, H., Hasuike, K., et al. 2006, PASJ, 58, L11

Uchiyama, Y., Aharonian, F. A., Tanaka, T., et al. 2007, Natur, 449, 576

Williams, B. J., Blair, W. P., Blondin, J. M., et al. 2011, ApJ, 741, 96

Williams, B. J., Hewitt, J. W., Petre, R., \& Temim, T. 2018, ApJ, 855, 118 